\title{
Forecasting of Ventricular Tachycardia using Scaling Characteristics and Entropy of Heart Rate Time Series
}

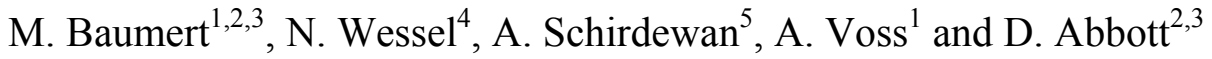 \\ ${ }^{1}$ Department of Medical Engineering, University of Applied Sciences Jena, Germany \\ ${ }^{2}$ Centre for Biomedical Engineering, The University of Adelaide, Australia \\ ${ }^{3}$ School of Electrical and Electronic Engineering, The University of Adelaide, Australia \\ ${ }^{4}$ Institute of Physics, University of Potsdam, Germany \\ ${ }^{5}$ Medical Faculty of the Charité, Franz Volhard Clinic, Helios Klinikum-Berlin, Germany
}

\begin{abstract}
In this paper we investigated heart rate time series stored by implantable cardioverter defibrillators in order to the short-term forecast ventricular tachycardia (VT). Standard heart rate variability (HRV) parameters, compression based entropy functions, and HRV scaling characteristics (detrended fluctuation analysis, Higuchi's fractal dimension) were analyzed in 29 VT time series and compared to individually acquired control time series. We found no differences in standard HRV parameters but significant changes in the entropy function as well as in the scaling characteristics of HRV before the onset of VT. In conclusion, HRV analysis might provide markers for early detection of forthcoming VT.
\end{abstract}

Keywords-Heart rate variability, compression entropy, scaling analysis, ventricular tachycardia

\section{INTRODUCTION}

Sudden cardiac death (SCD) is a major cause of mortality in the developed countries with an incidence of 3 million cases per year worldwide [1]. SCD is usually caused by a malignant tachyarrhythmia and implantable cardioverter defibrillators (ICDs) have been the most successful therapy in high risk patients [2]. Third generation ICDs are capable of storing the beat-to-beat intervals (BBIs) before VT, allowing and an assessment of the autonomous nervous system (ANS) before the onset of VT by means of heart rate variability (HRV). Since the ANS tone seems to have direct impact on the VT development we employ HRV several analysis techniques in order to forecast VT [3]. HRV analysis has been demonstrated to be a potential risk predictor in patients after myocardial infarction and is widely performed linearly in the time or frequency domains, as specified by the Task Force of the European Society of Cardiology and the North-American Society of Pacing and Electrophysiology [4]. Since numerous studies have pointed out the importance of complex HRV characteristics [5], we furthermore investigate the long-term correlations and the fractal dimension in heart rate time series by applying detrended fluctuation analysis (DFA) [6] and Higuchi's fractal dimension algorithm (HFD), respectively, in this study [7]. In addition, we estimate the HRV entropy with a novel approach based on data compression.

\section{MEthods}

\section{A. Data and preprocessing}

Fifty patients with severe congestive heart failure were enrolled at the Franz-Volhard-Hospital Berlin. No patient received a class I or III antiarrhythmic drug prior to the study. All patients had an implanted ICD (PCD 7220/ 7221 Medtronic) capable of storing 1024 BBI before the onset of a VT with a resolution of $10 \mathrm{~ms}$. HRV analysis of a VT time series is performed when there is fewer than 10 percent of ectopic beats/artifacts $(\mathrm{N}=29)$. These results are then compared to individually acquired and arbitrarily selected control time series (CON), without arrhythmic events that were stored just before a regular ICD follow-up examination (Fig. 1). Artifacts and ectopic beats were removed and interpolated by an algorithm using local variance estimation.

\section{B. Higuchi's fractal dimension}

To compute the fractal dimension of a graph, Higuchi [7] considers a finite set of observations $X(j), j=1,2, \ldots, N$ taken at a regular interval $k$, and evaluates the length $L_{\mathrm{m}}(k)$ of the corresponding graph for different interval lengths $k$ from sequence $X_{m}^{k}: \quad X(m), \quad X(m+k), \quad X(m+2 k), \quad \ldots$, $X\left(m+\left[\frac{N-m}{k}\right]\right)$, where $m=1,2, \ldots, k$ and $\left[\frac{N-m}{k}\right]$ denotes the integer part of $(N-m) / k$. The length of the graph is calculated as:

$$
L_{m}(k)=\left(\left[\sum_{i=1}^{\left.\frac{N-m}{k}\right] X(m+i k)-X(m+(i-1) k) \mid}\right) \frac{N-1}{\left[\frac{N-m}{k}\right] k^{2}} .\right.
$$

If the behavior of the graph has fractal characteristics over the available range $k$, then $L(k) \sim k^{-D f}$, where $D_{f}$ is the 
fractal dimension and $L(k)$ is the average value over $k$ partial lengths of the graph. For a straight line, $D_{f}=1$. For Brownian motion, $D_{f}=1.5$, and for Gaussian white noise, $D_{f}$ saturates at two. For time series with $1 / f^{\beta}$ power spectra, $D_{f}=(5-\beta) / 2$. This relationship is valid for $1<\beta<3$. Numerical experiments have shown that time series with the same $\beta$ can show different $D_{f}$ values, depending on the phase distribution [7].

\section{Detrended Fluctuation Analysis}

The DFA has been developed to analyze long-range correlations (long-memory dependence) in non-stationary data, where conventional fluctuation analyses such as power spectra and Hurst analysis cannot be reliably used [6]. The method works as follows:

1. Compute the cumulative sum $c(k)=\sum_{i=1}^{k}[s(k)-\bar{s}]$ of the time series $s$, where $\bar{s}$ is the mean of $S$ (using the concept of Random-Walk-Analysis).

2. Compute the local trend $c_{n}(k)$ within boxes of varying sizes $n$ (least square fit).

3. Compute the root mean square of the detrended time series in dependency on box size $n$ as $F(n)=\sqrt{\frac{1}{N} \sum_{k=1}^{N}\left[c(k)-c_{n}(k)\right]^{2}}$, where $N$ denotes the size of $S$.

4. Plot $\log _{10} F(n)$ against $\log _{10} n$.

If the data displays long-range dependence then $F(n) \sim$ $n^{\alpha}$, where $\alpha$ is the scaling exponent. For stationary data with scale-invariant temporal organization, the Fourier power spectrum $S(f)$ is $S(f) \sim f^{\beta}$, where the scaling exponent $\beta$ is related to $\alpha$ in the following way: $\beta=2 \alpha-1$. Values of $0<\alpha$ $<0.5$ are associated with anti-correlation (i.e. large and small values of the time series are likely to alternate). For Gaussian white noise $\alpha=0.5$. Values of $0.5<\alpha \leq 1$ indicate long-range power-law correlations (i.e. large values of the time series are likely to be followed by large values). Values $1<\alpha \leq 1.5$ represent stronger long-range correlations that are different from power-law, where $\alpha=1.5$ for Brownian motion. Scale-invariance in HRV has been commonly observed over a wide range with a characteristic break at segment sizes of 16 heart beats [6].

\section{Compression based entropy estimation}

In the framework of algorithmic information theory, the entropy, also called Kolmogorov-Chaitin complexity or algorithmic complexity, of a given text is defined as the smallest algorithm that is able to generate the text. Although it is theoretically impossible to develop such an algorithm, data compressors might represent a sufficient approxima- tion. We apply a modified version [8] of the LZ77 algorithm for lossless data compression [9]. The algorithm is based on a sliding window technique, looking for, and encoding, matching sequences between the window and a look ahead buffer. If the length $N$ of the text to be compressed is sufficiently large and the source is an ergodic process, the ratio of compressed text to original text length represents the 'compression' entropy $H_{c}$ per symbol. In this study, the look ahead buffer size is set sufficiently high and the window size is varied between one and 100. The decay of the entropy function $h_{\mathrm{c}} / d w$, depending on the window size provides information about auto-dependence.

\section{E. Standard HRV analysis}

For standard HRV analysis of VT and CON time series we calculate a parameter set of time and frequency domain measures (Tab.1) according to the HRV Task Force [4]. The frequency domain analysis is performed with linear interpolated time series of $500 \mathrm{~ms}$ resolution, using Fast Fourier Transform with Blackman-Harris windowing.

Table 1 Standard HRV parameter definitions.

\begin{tabular}{ll}
\hline Parameter & Definition \\
\hline meanNN & Mean of all normal NN intervals; in ms \\
sdNN & Standard deviation of all NN intervals; in ms \\
rmssd & RMS of successive NN interval differences; in ms \\
VLF & Power in the very low freq. band $(0.003-0.04 \mathrm{~Hz}) ;$ in $\mathrm{ms}^{2}$ \\
LF & Power in the low freq.band $(0.04-0.15 \mathrm{~Hz}) ;$ in $\mathrm{ms}^{2}$ \\
HF & Power in the high freq. band $(0.15-0.4 \mathrm{~Hz}) ;$ in $\mathrm{ms}^{2}$ \\
\hline LFn & Ratio of LF power to HF plus LF power \\
\hline
\end{tabular}

\section{F. Statistics}

To compare HRV parameters between CON and VT, we compute group means, standard deviations as well as Student's t-tests for paired data. Parameters were considered statistically significant, if $p<0.05$. Further, linear correlation coefficients are computed between HFD and DFA measures.

\section{Results}

To assess the fractal characteristics of HRV we relate the well-known VLF, LF, and HF frequency bands of HRV to the scaling graph, computing three different scaling exponents. For HFD, all three scaling exponents are significantly different from each other (ANOVA, $p<0.0001$ ). For DFA, ANOVA shows nearly significant differences between the three scaling exponents $(p=0.06)$. 
Table 2 Results of heart rate variability (HRV) analysis presented as means, standard deviations (SD) and paired Student's t-test results (p). n.s. - not significant. Residuals were multiplied by $10^{3}$ for clarity.

\begin{tabular}{|c|c|c|c|c|c|}
\hline \multirow[t]{2}{*}{ parameter } & \multicolumn{2}{|c|}{$\mathrm{CON}$} & \multicolumn{2}{|c|}{ VT } & \multirow[b]{2}{*}{$\mathrm{p}$} \\
\hline & mean & SD & mean & SD & \\
\hline meanNN & 769 & 142 & 709 & 154 & 0.06 \\
\hline sdNN & 48 & 31 & 43 & 32 & n.s. \\
\hline rmssd & 20 & 18 & 16 & 13 & n.s. \\
\hline VLF & 204 & 307 & 252 & 533 & n.s. \\
\hline LF & 80 & 158 & 86 & 247 & n.s. \\
\hline $\mathrm{HF}$ & 29 & 77 & 14 & 24 & n.s. \\
\hline LFn & 0.71 & 0.15 & 0.68 & 0.20 & n.s. \\
\hline$D_{f_{H F}}$ & 1.64 & 0.13 & 1.58 & 0.16 & 0.03 \\
\hline$D_{f_{\mathrm{LF}}}$ & 1.77 & 0.13 & 1.70 & 0.18 & 0.04 \\
\hline$D_{f_{\mathrm{VLF}}}$ & 1.83 & 0.21 & 1.85 & 0.31 & n.s. \\
\hline$r e s_{-} D_{f_{\mathrm{HF}}}$ & 1.49 & 1.17 & 1.68 & 1.41 & n.s. \\
\hline$r e s_{-} D_{f_{\mathrm{LF}}}$ & 0.34 & 0.14 & 0.36 & 0.18 & n.s. \\
\hline$r e s_{-} D_{f_{\mathrm{VLF}}}$ & 0.23 & 0.13 & 0.26 & 0.21 & n.s. \\
\hline$\alpha_{\mathrm{HF}}$ & 1.26 & 0.21 & 1.24 & 0.28 & n.s. \\
\hline$\alpha_{\mathrm{LF}}$ & 1.19 & 0.20 & 1.28 & 0.22 & 0.009 \\
\hline$\alpha_{\mathrm{VLF}}$ & 1.11 & 0.19 & 1.19 & 0.35 & n.s. \\
\hline$r e s \_\alpha_{\mathrm{HF}}$ & 3.00 & 1.22 & 3.00 & 1.30 & n.s. \\
\hline$r e s \_\alpha_{\mathrm{LF}}$ & 1.16 & 0.35 & 1.18 & 0.46 & n.s. \\
\hline$r e s \_\alpha_{\mathrm{VLF}}$ & 7.57 & 3.59 & 6.92 & 2.61 & n.s. \\
\hline$H_{\mathrm{c}} / d w$ & -0.078 & 0.023 & -0.086 & 0.027 & 0.03 \\
\hline$H_{\mathrm{c}}$ & 0.33 & 0.04 & 0.32 & 0.05 & n.s. \\
\hline
\end{tabular}

Post-hoc testing via Students' t-test indicates significant differences $(p=0.03)$ between the LF and VLF exponents of DFA, only. To test whether the scale-invariance, i.e. the fractal relationship, is less pronounced before the onset of VT, we compute the residuals (in Tab. 1 termed with the prefix 'res ') of the VLF, LF, and HF regression lines for HFD and DFA and find significant differences neither in HFD nor in DFA scaling exponent residuals. The exponents obtained via HFD show significant changes in the HF and LF range, being both decreased before the onset of VT. The scaling exponents obtained via DFA show significant changes in the LF band, only, indicating an increased longterm correlation before the onset of VT. Linear correlation coefficients between HFD and DFA are $r=-0.34$ for the HF range, $r=-0.69$ for the LF range, and $r=-0.57$ for the VLF range, respectively.

Compression based HRV analysis shows a significantly steeper initial decay of the entropy function with increasing window size. For group comparison, we compute the average decay $h_{\mathrm{c}} / d w$ for window length $1-3$. The entropy $H_{c}$ is computed over a range where the estimate becomes stable, i.e. over window size 90-100, and shows no changes before the onset of VT.
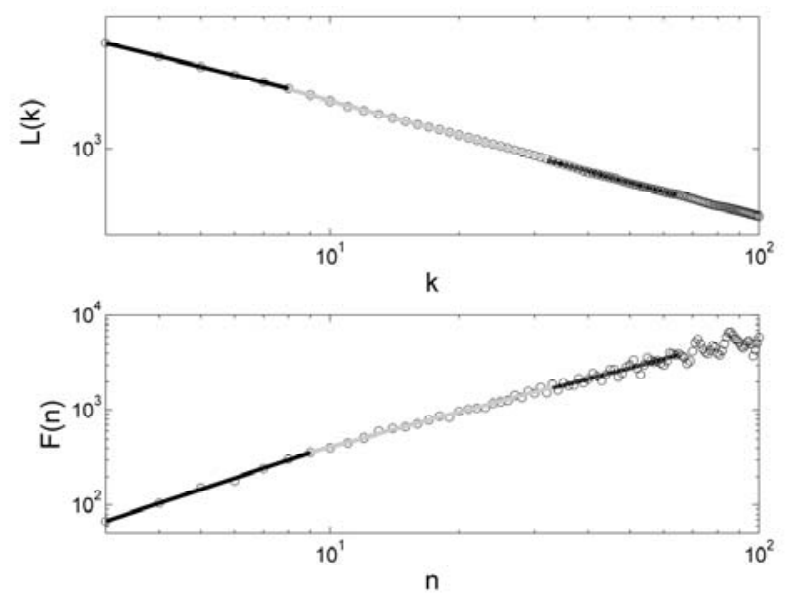

Fig. 1 Higuchi's fractal dimension analysis (top) and detrended fluctuation analysis (bottom) of one control heart rate time series. Black solid line high frequency (HF) scaling range. Gray solid line - low frequency (LF) scaling range. Back dotted line - very low frequency (VLF) scaling range.

None of the standard HRV parameters are significantly changed before the onset of VT.

\section{Discussion}

In this paper we analyze HRV before the onset of VT in order to derive markers for short-term forecasting. In accordance with other studies, we find no significant changes in the standard HRV parameters [10].

HFD analysis reveals three different fractal components of HRV. With larger scales the fractal dimensions increase. Before the onset of VT, we find decreased fractal dimensions, i.e. decreased roughness, in the HF and LF bands that might be understood as a general loss of short-term HRV.

DFA also reveals distinct fractal features of HRV. In $\mathrm{CON}$, long-term correlations decrease at larger scales. Before the onset of VT, we find increased long-term correlations in the LF band which is thought to reflect sympathetic and vagal influences [6] and is in agreement with other studies, suggesting increased sympathetic activity as one origin of arrhythmogenesis [11].

Considering the difference between HFD and DFA results and the moderate linear correlation, HRV cannot be fully explained as a self-affine stochastic process, where the local properties, assessed with HFD, reflect in the global properties, as assessed with DFA. Both methods should therefore be seen as two complementary approaches. The chosen method of adaptive scaling range computation is based on well-known physiological phenomena, traditionally considered in power spectrum analysis and demonstrating a good fit for all investigated recordings. Even though 
the three separate scaling exponents might not be fundamentally different in every case, they represent an intuitive, physiologically grounded way to assess the scaling graphs.

The analysis of the compression based entropy function indicates a loss of auto-dependence before VT. The significantly steeper decay of the entropy function indicates less complex structures, since the VT time series are comparatively better compressible at small window sizes. For large window sizes no significant differences occur, i.e. the entropy itself is not different before VT. Other studies, employing different complexity measures, have found less short-term fluctuations of HRV before the onset of VT, which is seen as a result of decreased vagal activity [1].

When employing HRV analysis for VT detection in ICDs, it has to be considered that only a subgroup of patients with ICDs exhibit a stable sinus rhythm with a limited number of ectopic beats. In our study, we analyze time series with less than 10 percent of ectopic beats/artifacts to make sure that HRV is not predominantly influenced by post-extrasystolic regulation patterns. Based on precise clinical diagnosis, future studies should therefore identify patho-physiological conditions that might allow reliable forecasting.

In conclusion, the compression based entropy function scaling features of heart rate time series are significantly changed before the onset of VT and might individually be suitable for short-term forecasting of life-threatening arrhythmias in ICDs.

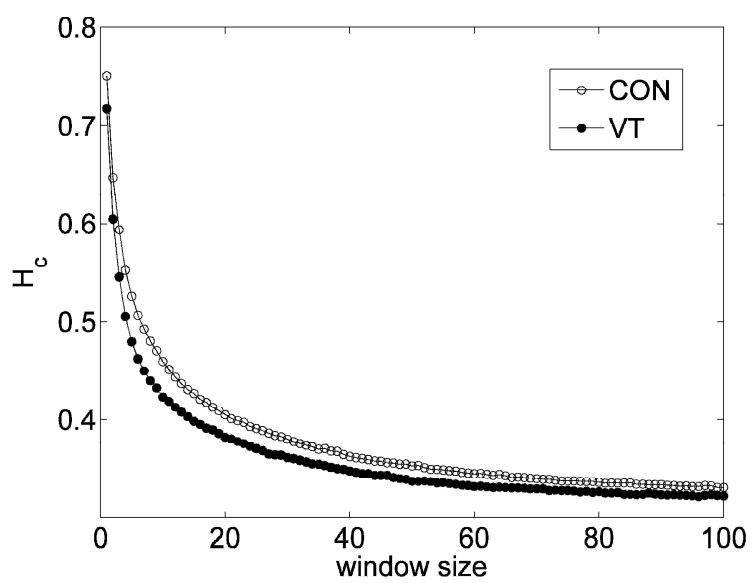

Fig. 2 Mean compression entropy of control heart rate time series (CON) and time series before VT as a function of the LZ77 compressor's window size.

\section{ACKNOWLEDGMENT}

This study was supported by grants form the Deutsche Forschungsgemeinschaft (DFG Vo505/3-1) and the Australian Research Council (DP0663345).

\section{REFERENCES}

1. Fogel RI, Prystowsky EN (2000) Management of malignant ventricular arrhythmias and cardiac arrest. Crit Care Med 28:165-169

2. Peters RW, Gold MR (2001) Implantable cardiac defibrillators. Med Clin North Am 85:343-367

3. Pruvot W, Thonet G, et al. (2000) Heart rate dynamics at the onset of ventricular tachyarrhythmias as retrieved from implantable cardioverter-defibrillators in patients with coronary artery disease. Circulation 23:2398-2404

4. Task Force of the European Society of Cardiology and the North American Society of Pacing and Electrophysiology (1996) Heart rate variability. Standards of measurement, physiological interpretation, and clinical use. Eur Heart J 17(3):354-831

5. Goldberger AL (1996) Non-linear dynamics for clinican: Chaos theory, fractals, and complexity at the bedside. Lancet 347:1312-1314

6. Peng CK, Havlin S, et al. (1995) Quantification of scaling exponents and crossover phenomena in nonstationary heartbeat time series. Chaos 5: 82-87

7. Higuchi $\mathrm{T}$ (1990) Relationship between the fractal dimension and the power law index for a time series: A numerical investigation. Physica D 46: 254-264

8. Baumert M, Baier V, et a. (2005) Estimating the complexity of heart rate fluctuations - An approach based on compression entropy. Fluct Noise Lett 5:L557-L563

9. Shannon CE (1948) A mathematical model of communication. The Bell System Technical J 27:379-423, 623-656

10. Vybiral T, Glaeser DH et al. (1993) Conventional heart rate variability analysis of ambulatory electrocardiographic recordings fails to predict imminent ventricular fibrillation. J Am Coll Cardiol 22: 557565

11. Lombardi F, Porta A, et al. (2000) Heart rate variability patterns before ventricular tachycardia onset in patients with an implantable cardioverter defibrillator. Participating Investigators of ICD-HRV Italian Study Group. Am J Cardiol 86: 959-963.

Address of the corresponding author:

$\begin{array}{ll}\text { Author: } & \text { Dr Mathias Baumert } \\ \text { Institute: } & \begin{array}{l}\text { School of Electrical \& Electronic Engineering } \\ \text { The University of Adelaide }\end{array} \\ & \text { SA 5005 } \\ \text { Country: } & \text { Australia } \\ \text { Email: } & \text { mbaumert@eleceng.adelaide.edu.au }\end{array}$

